\title{
Kapitel XI
}

Joanna Szczęk

Marcelina Kałasznik*

\section{Das Auge isst mit - Zu Schwierigkeiten im Bereich der deutsch-polnischen Übersetzung von kulinarischen Realien}

\section{Zusammenfassung}

Essen und Trinken sind nicht nur existenzielle Bedürfnisse des Menschen, sondern auch Identitätsträger und Elemente, mithilfe deren die jeweilige Kultur definiert wird. In Situationen, in denen man mit fremden Kulturen konfrontiert wird, stößt man oft auf Verstehensprobleme. Damit haben wir es auch, wenn nicht vor allem im Bereich des Kulinarischen zu tun. Im Mittelpunkt des Beitrags stehen Bezeichnungen für traditionelle deutsche Speisen und deren Übersetzungen ins Polnische. Die analysierten polnischen Übersetzungen entstammen Internetseiten und werden anschließend mit Wörterbuchäquivalenten verglichen. Aufgrund der Analyse und in Anlehnung an die Forschungsliteratur werden Strategien präsentiert, die beim Übertragen von kulinarischen Realienbezeichnungen eingesetzt werden.

Schlüsselwörter: Realien(bezeichnungen), Speisebezeichnungen, Kulinaristik, Übersetzung von Speisebezeichnungen, Übersetzung von Realien

\begin{abstract}
Eating and drinking are not only the existential human needs, but also carriers of identity and means by which a culture defines herself. In situations where confronted with foreign cultures, it is the field of culinary where one often encounters problems in understanding some of the content. In the center of this analysis are the names of traditional, German dishes and their translations into Polish. The analyzed Polish translations come from internet websites and are
\end{abstract}

${ }^{*}$ Dr. Joanna Szczęk (Uniwersytet Wrocławski), Marcelina Kałasznik, M.A. (Uniwersytet Wrocławski). 
then compared with the dictionary equivalents. Strategies will be presented on the basis of the analysis and in relation to the literature, which are used during translation of culinary "realia". Keywords: realia, culinary names, culinary studies, translation of culinary names, translation of realia

In Konfrontation mit anderen Kulturen hat man es oft mit Verstehensproblemen zu tun. Einer der Bereiche, in dem Schwierigkeiten oft anzutreffen sind, sind Kulinaria, mit deren Hilfe die jeweilige Kultur auch definiert wird ${ }^{1}$. Diese Tatsache wird auch in der einschlägigen Literatur hervorgehoben: „Speisen, Getränke und Mahlzeiten dienen im Alltag [...] zur Abgrenzung sozialer, ethnischer, religiöser Gruppen und Schichten. [...] Die Esskultur stellt ein starkes Element der kollektiven Identität dar" (Mannová 2007: 40). Von Feyrer (2009: 389) werden Kulinaria zu Universalien gezählt, und dies auf der Grundlage der Beobachtung, „dass wir Menschen uns über Kultur und Kulinaria definieren und Kulinaria als identitätsbildende Faktoren eine große Rolle im intra- und interkulturellen und sozialen Zusammenleben spielen."

Der starke Zusammenhang zwischen der menschlichen Existenz und dem Bereich des Kulinarischen verursacht, dass Kulinaria eine Herausforderung für Übersetzer darstellen, weil sie für das jeweilige Land charakteristische Zutaten und Produkte enthalten oder landesspezifische Gerichte benennen. Die Aufgabe der Übersetzer ist desto schwieriger, dass Kulinaria sowohl in der Forschungsliteratur ${ }^{2}$ als auch in der translatorischen Praxis ${ }^{3}$ oft stiefmütterlich behandelt werden (vgl. dazu Holzer 2009: 377, Lepenies 1997: 98). Kulinarisches ist in der heutigen Zeit nämlich nicht mehr als bloßes Essen und Trinken aufzufassen, sondern gehört zum Image eines jeden Landes und wie es Wierlacher (vgl. 2013: 635) angibt, sollten Essen und Trinken als „soziale Totalphänomene“ betrachtet werden, da Essen und Trinken nicht nur menschliche Grundbedürfnisse, sondern auch „[...] kulturtragende Kommunikationsformen, Handlungen, Situationen und Institutionen, Symbolsyste-

\footnotetext{
${ }^{1}$ Vgl. hierzu z.B. Barlösius/Naumann/Teuteberg (1996: 13-23), Neumann (1996: 385444), Feyrer (2009: 389), Tschofen (2008: 64), Holzer (2009: 377) u.a.

${ }^{2}$ Es lässt sich auf einige wissenschaftliche Arbeiten hinweisen, die das Thema des Übersetzens der Realien im Bereich der Kulinarien aufgreifen, vgl. z.B. Holzer (2009), Snell-Hornby (2008), Szczęk/Kałasznik (im Druck). Man kann jedoch die Tendenz beobachten, dass das Schwergewicht auf das Übersetzen und auf die (Un)Übersetzbarkeit der Realien in literarischen Texten gelegt wird, und das Übersetzen im Bereich des Kulinarischen eher trivial behandelt wird (vgl. dazu z.B. Snell-Hornby 2008).

${ }^{3}$ Davon zeugen z.B. die Speisekarten in den polnischen Restaurants, die weniger gelungene Übersetzungen der polnischen Speisenamen ins Deutsche enthalten. Aufgrund unserer Analyse der Fehler in polnisch-deutschen Übersetzungen der Speisebezeichnungen in polnischen Speisekarten können wir feststellen, dass die Übersetzungen nicht von professionellen Übersetzern angefertigt werden. Genaueres hierzu Szczęk/Kałasznik (im Druck).
} 
me, Rituale, Agrar-, Handels- und Industrieprodukte, Selbstbehauptungsmedien und Bestände der kulturellen Gedächtnisse" sind.

Aus dem Grunde sollte auch dem Übersetzen von kulinarischen Bezeichnungen, die als „Etiketten“ für landeskonventionelle Speisen fungieren, besondere Aufmerksamkeit geschenkt werden.

Im vorliegenden Beitrag werden deutsche Speisebezeichnungen in ihrer Übersetzung ins Polnische analysiert. Wir konzentrieren uns auf solche Speisenamen, die Träger typischer Realien für deutsche Kultur sind. ${ }^{4}$ Unser Augenmerk gilt der Auswertung der getätigten Übersetzungen. Anschließend werden die Übersetzungen mit Äquivalenten oder Erklärungen aus deutschpolnischen lexikographischen Werken konfrontiert. ${ }^{5}$ Zugleich wird versucht, die angewendeten Strategien bei der Übersetzung ${ }^{6}$ kultureller Inhalte in kulinarischen Namen zu präsentieren. Den Analysestoff liefern hierbei Internetseiten, die dem Thema Essen und Trinken gewidmet sind ${ }^{7}$.

Unser Beitrag gilt als der zweite Teil einer komplementär angelegten Analyse der Übersetzungen von Kulinarien in der Konfrontation des Sprachenpaars Deutsch und Polnisch. ${ }^{8}$

\section{Zu den kulinarischen Bezeichnungen}

An Speisebezeichnungen werden keine bestimmten Anforderungen gestellt. Die Nomination in diesem Bereich unterliegt fast keinen Regeln (vgl. Dąbrowska 1998), deswegen werden der Phantasie und Kreativität auf die-

${ }^{4}$ Aufgrund der Recherchen im Internet konnten wir eine Liste der Bezeichnungen für typisch deutsche, österreichische und schweizerische Speisen erstellen. Aus Raumgründen thematisieren wird in diesem Beitrag ausschließlich Speisenamen, die Träger typischer Realien für deutsche Kultur sind. Zu den Internetseiten, die wir auf charakteristisch deutsche Speisebezeichnungen durchsucht haben, siehe Quellenverzeichnis am Ende des Beitrags.

${ }^{5}$ Zur lexikographischen Darstellung von Realien vgl. z.B. Sieradzka (2011).

${ }^{6}$ Den Terminus der Übersetzungsstrategie verstehen wir als „bewusste Vorgehensweise“ beim Übersetzen eines Textes (vgl. Hejwowski 2004).

${ }^{7}$ Siehe Quellenverzeichnis am Ende des Beitrags. Kulinarische Internetseiten dienten uns zunächst als Quelle für die Suche nach Bezeichnungen für typisch deutsche Speisen, dann als Quelle für ihre Übersetzungen ins Polnische. Es handelt sich dabei um Übersetzungen, die auf polnischen kulinarischen Internetseiten zu finden sind, deren Ziel es ist, den Polen die deutsche Küche näher zu bringen. Es ist schwierig, im Falle dieser Internetseiten ihren Adressatenkreis exakt zu bestimmen. Es kann angenommen werden, dass kulinarische Internetseiten sich an alle Küchen-Interessierten richten können, wobei sowohl ihr Wissensstand als auch ihre Kochkenntnisse ganz unterschiedlich sein können. Wie man sehen kann, sind die Übersetzungen auch unterschiedlichen Textsorten entnommen. Hierbei handelt es sich erstens um Kochrezepte für typisch deutsche Speisen, in denen sowohl ihre Originalbezeichnungen als auch die Übersetzungen ins Polnische erwähnt werden, Blogbeiträge, Kommentare usw.

${ }^{8}$ Vgl. Szczęk/Kałasznik (im Druck). 
sem Gebiet keine Grenzen gesetzt (vgl. Schirrmeister 2000: 133). Die Speisebezeichnungen erfüllen nicht nur die nominative Funktion, sondern vor allem die pragmatische: „Worte und veritable Fachsprachen, man denke an das Weinvokabular, erwecken semantische Verweise, assoziieren Welten, die das tatsächliche Essen auf dem Teller symbolisch übertragen“ (ebd.: 132f.).

Speisebezeichnungen sind in den meisten Fällen durch ihre ausgebaute Struktur gekennzeichnet. Formal gesehen ist die Mehrheit der Speisebezeichnungen analytisch, synthetische Namen sind nur gelegentlich anzutreffen.

Eine umfassende Typologie der Speisebezeichnungen ${ }^{9}$ nach dem semantischen Kriterium hat Witaszek-Samborska (2005: 123-163) vorgelegt. In dieser Aufteilung zerfallen Speisebezeichnungen in folgende Gruppen:

1. Namen für die Zutaten eines Gerichts, z.B. Nusstorte mit Ananas;

2. Namen, bei denen die Zubereitungsart eines Gerichts hervorgehoben wird:

a) Namen, welche die technischen Aspekte der Zubereitung eines Gerichts beschreiben, z.B. garnierte Eier,

b) Namen, welche die pragmatischen Aspekte der Zubereitung eines Gerichts beschreiben, z.B.: Bohnen auf alte Art;

3. Genetische Namen:

a) ethno-geographische Namen, z.B.: Krakauer Quarkkuchen, Bamberger Teller,

b) Namen, die von den Namen sozialer Schichten abgeleitet werden, z.B.: Jägerpastete,

c) familiäre Bezeichnungen, z.B.: Großmutterkuchen,

d) Namen, die zum Andenken an den Autor oder Patron des Gerichts gegründet wurden, z.B.: Stroganow;

4. Sensorische Namen:

a) positiv wertende Namen, z.B.: Luxuspastete,

b) Namen, welche sich auf die Geschmackseindrücke beziehen, z.B.: schmackhafter Braten,

c) Namen, welche sich auf die Sichteindrücke beziehen, z.B.: bunter Auflauf,

d) Namen, welche sich auf die Tasteindrücke beziehen, z.B.: gefrorene Schokolade,

e) Namen, welche sich auf die Geruchseindrücke beziehen, z.B.: duftender Tee,

f) Namen, welche sich auf die Gehöreindrücke beziehen, z.B.: knusprige Brötchen;

${ }^{9}$ Die Typologie von Witaszek-Samborska (2005) umfasst zwar nur Speisebezeichnungen aus dem Polnischen. Unsere früheren Analysen bestätigen jedoch, dass sie sich auch für die deutschen Bezeichnungen aus dem analysierten Bereich anwenden lässt. 
5. Namen, welche die Funktion, Bestimmung und Verwendung eines Gerichts beschreiben:

a) temporale und Gelegenheitsnamen, z.B.: Frühlingssuppe,

b) Funktionsnamen, z.B.: Spinat für Teigtaschen,

c) Namen mit Adressatenbezug, z.B.: Lachs für Verliebte;

6. Namen, welche die Wirkung des Gerichts auf den Konsumenten beschreiben. ${ }^{10}$

Die umfangreiche semantische Typologie verweist auf die Vielfalt im Bereich der kulinarischen Namengebung. Die im Folgenden analysierten Speisebezeichnungen, die gleichzeitig als deutsche Realienbezeichnungen fungieren, sind jedoch hinsichtlich ihrer Struktur und Semantik nicht so stark differenziert. Es handelt sich dabei vorwiegend um synthetische Namen, was die von Żarski (vgl. 2003: 161)in Bezug auf Suppennamen aufgestellte These zu bestätigen scheint, dass Ein-Wort-Bezeichnungen am häufigsten Bezeichnungen für Gerichte sind, die in einer Sprachgemeinschaft als traditionell empfunden werden.

\section{Zum Realienbegriff}

Realien ${ }^{11}$ finden in der übersetzungswissenschaftlichen Literatur oft Berücksichtigung, da sie als Elemente gelten, bei denen die Frage nach der (Un) Übersetzbarkeit besonders deutlich zum Tragen kommt.

Realien werden „[...]als kulturspezifische und deshalb fremde Träger von Lokalkolorit aufgefasst, die als Teilmenge lexikalischer Lücken für den Übersetzer Übersetzungsprobleme wegen Äquivalenzproblemen darstellen" (Kujamäki 1998: 17, Hervorhebungen im Original). Holzer unterstreicht ebenfalls den starken Zusammenhang zwischen Realien und Kultur, indem er sie als „[...] Identitätsträger eines nationalen oder ethnischen Gebildes bzw. einer nationalen oder ethnischen Kultur [...]" bezeichnet (Holzer 2009: $378)^{12}$. Die starke Kulturgebundenheit und feste Verankerung in bestimmter Sprachgemeinschaft hat zur Folge, dass Realien „[...] kaum eine Ent-

${ }^{10}$ Vgl. dazu Szczęk/Kałasznik (2012).

${ }^{11}$ In der Fachliteratur funktionieren auch andere Begriffe für dieses Phänomen, z.B. Schlüsselbegriffe/ Schlüsselszenarien, Kultureme, Kulturspezifika, kulturgebundene Elemente.

${ }^{12}$ Vgl. Hierzu auch die Explikation von Stanaszek: „te elementy (fragmenty) rzeczywistości opisywanej przez dany język, które albo nie istnieją w rzeczywistości obsługiwanej przez inny język, albo owszem istnieją, ale pełnią inną funkcję, czy też wyraźnie inaczej są postrzegane [...]” (2007: 449). Übersetzung des Zitats ins Deutsche- M.K.: „diese Elemente (Fragmente) der von einer Sprache beschriebenen Wirklichkeit, die es entweder in der von einer anderen Sprache bedienten Wirklichkeit nicht gibt oder die es gibt, die aber eine andere Funktion erfüllen oder deutlich anders wahrgenommen werden“. 
sprechung bei anderen Völkern, in anderen Ländern oder an anderen Orten haben" (Chojnowski 2001: 24). Kujamäki (2004: 920) teilt Realien in zwei Untergruppen auf: 1. „Dinge einer bestimmten Klasse, etwa Tierarten, Sitten und Gebräuche, Speisen und Getränke" und 2. Realien, die Einzeldinge bezeichnen, z.B. Bergnamen, Bezeichnungen von bestimmten Behörden usw. Mędelska/Wawrzyńczyk (1992: 34, zit. in Chojnowski 2001) unterscheiden nach dem Kriterium des Bezeichneten „Namen für Feiertage, Bräuche, Spiele, Bezeichnungen von Gegenständen, Kleidern, Speisen, Getränken sowie Namen von Organisationen, ihren Mitgliedern, Berufen“. In Bezug auf Kulinarien wird somit darauf hingewiesen, dass sie ein Teil der Realien sein können (vgl. Stanaszek 2007: 449). Sie werden in der Forschungsliteratur zu ethnographischen Realien gezählt (vgl. Armalytė 1986: 9f., Vlachov/Florin 1980: 52ff., zit. in Kohrs 2008: 62). Mit Speisebezeichnungen können also in verschiedenen Trägern von Kulinarien, in der Literatur, in der Presse usw. Kulturbezüge geschaffen werden. In Anlehnung an die Typologie von von Kulturbezügen ${ }^{13}$ von Gercken (1999: 113f.) kann festgestellt werden, dass es sich dabei um explizite Kulturbezüge handelt, die sich durch kulturtragende Lexeme oder Eigennamen manifestieren, die bestimmte kulturspezifische Bezeichnungsinhalte benennen. Das bestätigt auch Wierzbicka (2007: 16), die die Lexik des Essens und Trinkens als das prägnanteste und offensichtlichste Beispiel für den Zusammenhang zwischen dem Wortschatz einer Sprache und der Kultur deren Sprecher aus dem Bereich des Materiellen betrachtet.Aus dem Grunde stellen Kulinarien potentielle Übersetzungsprobleme dar, die sprachextern und kulturbedingt sind.

\section{Analyse des Materials}

In unserer Analyse konzentrieren wir uns auf kulinarische Bezeichnungen, die Träger der deutschen nationalen und regionalen Identität sind. Die von uns untersuchten Speisenamen benennen einerseits typisch deutsche Speisen, die sowohl von Ausländern als auch von Deutschen selbst so eingestuftwerden. Sie stellen zugleich eine potentielle Übersetzungsschwierigkeit ${ }^{14}$ dar, $^{2}$ die folgendermaßen definiert wird: „Eine Übersetzungsschwierigkeit liegt überall dort, wo ausgangssprachliches Textverständnis vorausgesetzt, eine lexikalische, syntagmatische oder syntaktische Eins-zu-Eins-Entsprechung zwischen ausgangs- und zielsprachlichem Textsegment nicht möglich ist und substitutive Übersetzungsprozeduren eindeutig registrierbare sprachliche Fehlleistungen zur Folge hätten“" (Koller 1994: 35f.).

${ }^{13}$ Gercken (1999) differenziert zwischen expliziten und impliziten Kulturbezügen.

${ }^{14}$ Zur Typologie von Übersetzungsschwierigkeiten vgl. Thome (2004). 
Im Folgenden werden folgende Speise bezeichnungen und deren Übersetzungen ins Polnische analysiert: Currywurst, Eintopf, Himmel und Erde, kalter Hund, Kass(e)ler, Königsberger Klopse, Sauerbraten, Schupfnudeln, Spätzle ${ }^{15}$.

\subsection{Analyse der Übersetzungen}

In der nachfolgenden Tabelle wird jeweils erklärt, was für eine Speise sich hinter dem Namen versteckt. Die Erläuterungen, woraus und wie eine Speise zubereitet wird, können bei der Bestimmung der Vorgehensweise beim Übersetzen behilflich sein und einen Beitrag zur Charakteristik der erwähnten Küche leisten. Die angeführten Beispiele wurden in unveränderter Form Internetseiten entnommen. Die gefundenen, jedoch in vielen Fällen fehlerhaften Übersetzungen wurden nicht verbessert und mit den Äquivalenten aus den deutsch-polnischen Wörterbüchern konfrontiert.

Tabelle 1. Analyse der Übersetzungen von ausgewählten deutschen kulinarischen Realien ins Polnische

\begin{tabular}{|c|c|}
\hline Deutsche Speisebezeichnung & Übersetzungen ins Polnische ${ }^{*}$ \\
\hline \multicolumn{2}{|l|}{ Currywurst } \\
\hline \multicolumn{2}{|c|}{$\begin{array}{l}\text { Bedeutung: mit Curry bestreute, mit einer Currysoße oder Ketchup übergossene Brat } \\
\text { wurst }^{1}\end{array}$} \\
\hline Currywurst & $\begin{array}{l}\text { Currywurst } \\
\text { Kiełbasa w sosie curry } \\
\text { Niemieckie kietbaski w sosie curry }\end{array}$ \\
\hline \multicolumn{2}{|c|}{$\begin{array}{l}\text { Wörterbuchäquivalente: } \\
\text { IP (1987): kein Eintrag } \\
\text { PWN (2010): pieczona kiełbasa z sosem z curry } \\
\text { PONS (2007): kiełbasa z curry }\end{array}$} \\
\hline \multicolumn{2}{|l|}{ Eintopf } \\
\hline $\begin{array}{l}\text { Bedeutung: einfaches Gericht aus Ge } \\
\text { Zutaten zusammen in einem Topf gek }\end{array}$ & $\begin{array}{l}\text { artoffeln o.Ä. [und Fleisch], bei dem alle } \\
\text { rden; Kurzform: Eintopf }{ }^{4}\end{array}$ \\
\hline
\end{tabular}

${ }^{15}$ Die von uns genannten Beispiele für Bezeichnungen, die für Deutschland typische Speisen benennen, schöpfen jedoch die Liste aller solchen Bezeichnungen nicht aus. 


\begin{tabular}{|c|c|}
\hline $\begin{array}{l}\text { Eintopf } \\
\text { Eintopfgericht }\end{array}$ & $\begin{array}{l}\text { Eintopf }^{5} \\
\text { Zupa eintopf } \\
\text { Danie jednogarnkowe }\end{array}$ \\
\hline \multicolumn{2}{|c|}{$\begin{array}{l}\text { Wörterbuchäquivalente: } \\
\text { IP (1987): obiad jednodaniowy } \\
\text { PWN (2010): danie jednogarnkowe } \\
\text { PONS (2007): danie jednogarnkowe }\end{array}$} \\
\hline \multicolumn{2}{|l|}{ Himmel und Erde } \\
\hline \multicolumn{2}{|c|}{$\begin{array}{l}\text { Bedeutung:ein traditionelles rheinisches, westfälisches, niedersächsisches und schlesi- } \\
\text { sches Gericht aus Stampfkartoffeln und Apfelmus. Im Rheinland nimmt man traditionell } \\
\text { frische Kartoffeln und frische Äpfel, die gewürfelt, gekocht, aber keinesfalls gestampft } \\
\text { werden; Gebratene Blutwurstscheiben, Apfelmus und Kartoffelpüree machen den Sonn- } \\
\text { tagsklassiker Himmel und Erde aus. „Erde“ steht für Kartoffeln und „Himmel“ für die Äpfel } \\
\text { vom Baum }\end{array}$} \\
\hline Himmel und Erde & Niebo i ziemia ${ }^{9}$ \\
\hline \multicolumn{2}{|c|}{$\begin{array}{l}\text { Wörterbuchäquivalente: } \\
\text { IP (1987):kein Eintrag } \\
\text { PWN (2010): ,potrawa składająca się z ziemniaków, jabłek, smażonej kaszanki i wątrobi- } \\
\text { anki' } \\
\text { PONS (2007): kein Eintrag }\end{array}$} \\
\hline \multicolumn{2}{|l|}{ Kalter Hund } \\
\hline \multicolumn{2}{|c|}{$\begin{array}{l}\text { Bedeutung:ein kalt zubereiteter Kastenkuchen aus Schichten von Schokoladenmasse und } \\
\text { Butterkeksen }{ }^{10}\end{array}$} \\
\hline Kalter Hund & Zimny pies ${ }^{11}$ \\
\hline \multicolumn{2}{|c|}{$\begin{array}{l}\text { Wörterbuchäquivalente: } \\
\text { IP (1987):kein Eintrag } \\
\text { PWN (2010):rodzaj ciasta z warstw ciastek i masy kakaowej } \\
\text { PONS (2007): kein Eintrag }\end{array}$} \\
\hline \multicolumn{2}{|l|}{ Kassler } \\
\hline \multicolumn{2}{|c|}{$\begin{array}{l}\text { Bedeutung:gepökeltes und geräuchertes Schweinefleisch von Rippe, Kamm, Schulter } \\
\text { oder Bauch }{ }^{12}\end{array}$} \\
\hline $\begin{array}{l}\text { Kassler } \\
\text { Kasseler }\end{array}$ & $\begin{array}{l}\text { Baleron } \\
\text { Pieczony schab wędzony }{ }^{13} \\
\text { Schab peklowany }{ }^{14} \\
\text { Peklowane } \text { i wędzone mięso } \text { wieprzowe }^{15} \\
\text { Kotlety wędzone }^{16} \\
\text { Kotlety ze schabu }_{\text {Kassler }^{17}} \\
\text { Szynka kassler }{ }^{18}\end{array}$ \\
\hline
\end{tabular}




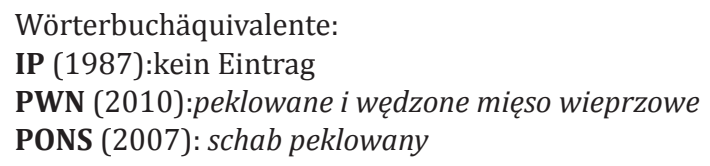

Königsberger Klopse

Bedeutung:edle Frikadellen in einer Sahne-Kapern-Sauce ${ }^{19}$; Königsberger Klopse ist ein traditionelles altes deutsches Rezept, bei dem die Klopse aus Hackfleisch in einer leicht säuerlichen Mehlschwitze serviert werden ${ }^{20}$

\begin{tabular}{|l|l|}
\hline $\begin{array}{l}\text { Königsberger Klopse } \\
\text { Kapern-, Soß- oder Kochklopse }\end{array}$ & Klopsy w sosie warzywnym ${ }^{21}$ \\
\hline Wörterbuchäquivalente: & \\
IP (1987):kein Eintrag & PWN (2010): kein Eintrag \\
PONS (2007): klopsiki duszone w sosie kaparowym
\end{tabular}

Sauerbraten

Bedeutung:in Essig mit Gewürzen marinierter und geschmorter Rinderbraten ${ }^{22}$ Sauerbraten Bawarska pieczeń z marynowanego mięsa ${ }^{23}$

Wörterbuchäquivalente:

IP(1987): pieczeń w occie moczona

PWN (2010): pieczeń na dziko

PONS (2007): pieczeń wołowa w marynacie

Schupfnudeln

Bedeutung: kleine rollenförmige Nudeln mit spitzen Enden. Für den Teig der Schupfnudeln werden Kartoffeln gekocht, gepresst und mit Weizenmehl, Butter und Eiern verarbeitet ${ }^{24}$.

Schupfnudeln

Fingernudeln
Szare kluski ${ }^{25}$

Kluski ziemniaczane ${ }^{26}$

Niemieckie kluski ziemniaczane ${ }^{27}$

Kluski

Wörterbuchäquivalente:

IP (1987): kein Eintrag

PWN (2010): kein Eintrag

PONS (2007): kein Eintrag

Spätzle

Bedeutung: kleine, längliche Stücke aus Nudelteig, die in siedendem Salzwasser gekocht werden28 


\begin{tabular}{|c|c|}
\hline $\begin{array}{l}\text { Spätzle } \\
\text { Spätzli } \\
\text { Knöpfle }\end{array}$ & $\begin{array}{l}\text { Szpecle }^{29} \\
\text { Zacierki }^{30} \\
\text { Spatzle }^{31} \\
\text { Mączne kluseczki }^{32}\end{array}$ \\
\hline \multicolumn{2}{|c|}{$\begin{array}{l}\text { Wörterbuchäquivalente: } \\
\text { IP (1987): rodzaj klusek } \\
\text { PWN (2010:):zacierki, kluski } \\
\text { PONS (2007): zacierki ('Szwabska specjalność przygotowywana z ciasta, które rozwał- } \\
\text { kowuje się cienko na stolnicy. Następnie cienkie paski ciasta wrzuca się do wrzącej wody i } \\
\text { gotuje') }\end{array}$} \\
\hline
\end{tabular}

* Die gesammelten Beispiele lassen den Schluss zu, dass nicht nur Realienbegriffe sondern auch Speisebezeichnungen überhaupt (trotz ihrer Transparenz) Übersetzenden Schwierigkeiten bereiten. Die genaue Erörterung der Fehler in präsentierten Übersetzungen würde den Rahmen dieses Beitrags sprengen. Genaueres zu Fehlern in Übersetzungen von Speisekarten vgl. Szczęk/Kałasznik (im Druck).

Quellen:

${ }^{1} \mathrm{http}$ //universal_lexikon.deacademic.com/158116/Curryso\%C3\%9Fe, Zugriff am 22.09.2014.

${ }^{2}$ http://iinspiracje-kulinarne.blogspot.com/2013/12/currywurst-cos-z-kuchni-niemieckiej.html, Zugriff am 15.05.2014.

${ }^{3}$ http://www.przyslijprzepis.pl/przepis/currywurst-czyli-niemieckie-kielbaskie-zcurry, Zugriff am 15.05.2014.

${ }^{4}$ http://www.duden.de/rechtschreibung/Eintopfgericht, Zugriff am 7.05.2014.

${ }^{5}$ http://www.gotowanie.v9.pl/slownik/66.php, Zugriff am 15.05.2014.

${ }^{6}$ http://ugotuj.to/ugotuj/-1797869/zupa+eintopf+po+wielkopolsku/p/, Zugriff am 15.05.2014.

${ }^{7}$ http://smaker.pl/polecane/Eintopf, Zugriff am 15.05.2014.

${ }^{8}$ http://www.essen-und-trinken.de/himmel-und-erde, Zugriff am 8.05.2014.

${ }^{9}$ http://www.facetnatalerzu.pl/2012/03/niebo-i-ziemia-czyli-himmel-und-erde.html, Zugriff am 15.05.2014.

${ }^{10}$ http://www.essen-und-trinken.de/kalter-hund, Zugriff am 7.05.2014.

11 http://kwiecienkaodkuchni.blox.pl/2011/02/Kalter-Hund.html, Zugriff am 15.05.2014.

${ }^{12}$ http://www.duden.de/rechtschreibung/Kassler_Fleisch_gepoekelt, Zugriff am 7.05.2014.

${ }^{13}$ http://zmiksowani.pl/przepisy-na/kasseler-pieczony-schab-wedzony, Zugriff am 15.05.2014.

14 http://www.forumowisko.pl/topic/54173-niemiecki/, Zugriff am 15.05.2014.

15 http://www.magicwindow.internetdsl.pl/dok/niemcy.pdf, Zugriff am 15.05.2014.

${ }^{16}$ http://bistro24.pl/gotowanie/przepisy/kotlety-wedzone-kassler-w-glazurze-miodowej,116819.html, Zugriff am 15.05.2014.

${ }^{17} \mathrm{http} / /$ /www.grupy.senior.pl/Re-OT-Nazewnictwo-kulinarne,p,2318462,f,1,9.html, Zugriff am 15.05.2014.

${ }^{18}$ http://barbaramariawkuchni.blogspot.com/2013/01/szynka-kassler-z-zapiekanka-ziemniaczana.html, Zugriff am 15.05.2014.

${ }^{19}$ http://www.essen-und-trinken.de/koenigsberger-klopse, Zugriff am 7.05.2014.

20 http://www.marions-kochbuch.de/rezept/0102.htm, Zugriff am 7.05.2014. 
${ }^{21}$ http://durszlak.pl/przepisy-kulinarne/klopsy-w-sosie, Zugriff am 15.05.2014.

${ }^{22}$ http://www.duden.de/rechtschreibung/Sauerbraten, Zugriff am 7.05.2014.

${ }^{23}$ http://allrecipes.pl/przepis/3769/bawarska-piecze--z-marynowanego-mi-sa--sauerbraten-.aspx, Zugriff am 15.05.2014.

${ }^{24}$ http://www.essen-und-trinken.de/schupfnudeln/schupfnudeln-1008432.html?eid=1008459, Zugriff am 7.05.2014.

${ }^{25}$ http://hasla.blox.pl/Blox/szukaj/kluski+z+ziemniakami, Zugriff am 15.05.2014.

${ }^{26}$ http://zmiksowani.pl/przepis,567900,kluski-ziemniaczane-badische-schupfnudeln. html, Zugriff am 15.05.2014.

27 http://kobieta.interia.pl/kuchnia/makarony/news-schupfnudeln-niemieckie-kluski-ziemniaczane,nId,709211, Zugriff am 15.05.2014.

${ }^{28}$ http://www.duden.de/rechtschreibung/Spaetzle, Zugriff am 7.05.2014.

${ }^{29}$ http://www.facetnatalerzu.pl/2012/01/spatzle-szpecle-maczne-kluseczki.html, Zugriff am 15.05.2014.

${ }^{30}$ http://www.pieceofcake.pl/Przepisy/Zacierka, Zugriff am 15.05.2014.

31 http://www.mojewypiekiinietylko.com/2014/07/szpecle-spatzle/, Zugriff am 15.05.2014.

${ }^{32}$ http://www.facetnatalerzu.pl/2012/01/spatzle-szpecle-maczne-kluseczki.html

\subsection{Zu den Übersetzungsstrategien von kulinarischen Realien aus dem Deutschen ins Polnische ${ }^{16}$}

Im Folgenden wird versucht, aufgrund der angeführten Beispiele eine Typologie der Strategien für deutsch-polnische Übersetzung der gesammelten Namen zu erstellen. In Anlehnung an Koller (2004: 228) liegt in diesem Kontext die Eins-zu-Null-Entsprechung vor, da in der Zielsprache durch die in der Ausgangssprache gebrauchten Realienbezeichnungen Lücken entstehen. Stanaszek (2007: 455) zufolge kann man jedoch feststellen, dass „[...] w przypadku realiów z natury rzeczy istnieje więcej możliwości ich przełożenia niż w przypadku pojęć nie będących realiami"17. Stanaszek (ebd.) schlägt in diesem Zusammenhang folgende Strategien für die Übersetzung von Realienbezeichnungen vor:

- Übertragung der Bezeichnung in Originalform;

- Übertragung der Bezeichnung in Originalform und Hinzufügen von Erklärungen;

- Modifikation der Bedeutung oder ihrer übersprachlichen Form - Ersetzen der Originalbezeichnung durch die Bezeichnung aus der Zielsprache;

- Nicht-Übertragung der Bedeutung der Bezeichnung in keinerlei Hinsicht und Form.

${ }^{16}$ Vgl. dazu auch Szczęk/Kałasznik (im Druck).

17 „Im Falle der Realien gibt es von Natur aus mehr Möglichkeiten deren Übertragung als im Falle von Begriffen, die keine Realien sind." (Übersetzung ins Deutsche - M.K.) 
Kujamäki(vgl. 1998: 78-88) weist in diesem Fall auf folgende Strategien hin:

- Fremdwortübernahme - Übernahme des ausgangssprachlichen Wortes als Zitatwortes;

- Lehnübersetzung, d.h. Glied-für-Glied-Übersetzung, die vor allem bei „sprechenden“ Realienbezeichnungen angewendet werden kann;

- erklärendes Übersetzen - der AS-Ausdruck wird mit einer Beschreibung versehen;

- Anwendung einer Analogie in der Zielsprache - Einsatz eines am nächsten liegenden Äquivalents. In diesem Fall geht ein Teil der Kulturspezifik des AS-Textes unter;

- hyperonymische Übersetzung - Einsatz eines semantisch übergeordneten Begriffs;

- kohyponymische Übersetzung - Einsatz eines Kohyponyms;

- assoziative Übersetzung - Vorgehensweise, „[...] die sich betont nach der kontextuellen, oft übertragenenBedeutung des Ausdrucks richtet, fast ohne jede lexikalisch-formale Anlehnung an das ausgangssprachliche Vorbild [...]" (Kujamäki1998: 87);

- Auslassung;

- Hinzufügung.

Koller (vgl. 2004: 232 ff.) schlägt in diesem Zusammenhang fünf Verfahren vor, um die lexikalischen Lakunen in der ZS zu schließen:

1. Übernahme des AS-Ausdrucks in die ZS (ggf. in Anführungszeichen), darunter Zitat- und Lehnwort;

2. Lehnübersetzung;

3. Einsatz eines am nächsten liegenden Äquivalents;

4. Explikation oder definitorische Umschreibung;

5. Adaptation.

Betrachtet man die angeführten Typologien, kann festgestellt werden, dass sie sich in den meisten Punkten decken.

In Anlehnung an die oben dargestellten ausgewählten Typologienund im Lichte des untersuchten Korpus lassen sich folgende Strategien beim Übersetzen von kulinarischen Bezeichnungen mit Komponenten, die typisch für die deutsche Küche sind, ins Polnische unterscheiden:

A. Übernahme des AS-Ausdrucks in die ZS (ggf. in Anführungszeichen):

a) Zitatwort, z.B.: Currywurst $\rightarrow$ Currywurst, Eintopf $\rightarrow$ Eintopf, Kassler $\rightarrow$ Kassler;

b) Lehnwort, z.B.: Spätzle $\rightarrow$ Szpecle;

B. Lehnübersetzung, z.B.: Himmel und Erde $\rightarrow$ Niebo i ziemia, Kalter Hund $\rightarrow$ Zimnypies;

C. Einsatz eines am nächsten liegenden Äquivalents, z.B.: Schupfnudeln $\rightarrow$ szare kluski, Spätzle $\rightarrow$ zacierki, mączne kluseczki; 
Explikation oder definitorische Umschreibung, z.B. Kassler $\rightarrow$ peklowane i wędzone mięso wieprzowe, Himmel und Erde $\rightarrow$,potrawa składająca się z ziemniaków, jabłek, smażonej kaszanki i wątrobianki'.

\section{Schlussfolgerungen}

Der Bereich des Kulinarischen, obwohl er häufig als trivial betrachtet wird und in der translatorischen Praxis eher stiefmütterlich behandelt wird, erweist sich im Kontext derÜbersetzung von kulinarischen Realien als eine schwierig zu bewältigende Aufgabe. Im Falle der Realienbezeichnungen aus dem Gebiet des Kulinarischen ist die Glied-für-Glied-Übersetzung, die sich bei anderen Speisebezeichnungen in vielen Fällen anwenden lässt, weil sie aus Appellativen bestehen, ausgeschlossen. Die Undurchsichtigkeit der Namen erzwingt andere Techniken und Strategien, zu denen der Übersetzer greifen muss, um sich mit dieser translatorischen Herausforderung auseinandersetzen zu können. Aufgrund unserer Typologie von Übersetzungsstrategien, -vorschlägen und -lösungen wagen wir keine quantitativen Schlussfolgerungen zu ziehen, da die Anzahl der untersuchten Beispiele zu gering ist, um übergreifende Schlüsse im Hinblick auf den Einsatz von einzelnen Strategien zu formulieren.

Es lässt sich jedoch sagen, dass Übersetzende gerne die Realienbezeichnungen aus der AS übernehmen. Pragmatisch gesehen erweist sich diese Strategie als günstig, wenn man weiß, dass der Rezipient mit der Zielkultur vertraut ist. Sonst wird der Name als etwas Fremdes wahrgenommen und erweckt keine Assoziationen.

Es lassen sich auch Beispiele für die Glied-für-Glied-Übersetzung anfinden. Solche Übersetzungen betreffen jeweils zweigliedrige Speisebezeichnungen, die aus appellativischen Elementen bestehen. Man muss jedoch anmerken, dass der kommunikative Wert solcher Übersetzung auch nicht zufriedenstellend ist, da sie ebenfalls keine Informationen über die Speise liefern. Im Deutschen handelt es sich hier um lexikalisierte Verbindungen, deren wörtliche Übersetzungen ihre Funktion einfach nicht erfüllen können.

Die weitere Strategie, die in dem Einsatz des am nächsten liegenden Äquivalents besteht, hat ihre Vorteile. Hier wird auf etwas Ähnliches oder Vergleichbares aus der Zielkultur hingewiesen, wodurch sich der Rezipient ein Bild von der Speise machen kann. In diesem Sinne sind die Übersetzungen informativ, obwohl die Kulturgebundenheit der Bezeichnungen in den Hintergrund rückt.

Bei der Explikation wird ganz von der Speisebezeichnung abgesehen. Die Speise wird beschrieben, so dass der Rezipient weiß, was er erwarten kann. 
Die Beschreibungen ähneln in vielen Fällen den Kochrezepten. Diese Strategie lässt sich auch mit der Übernahme des fremden Lexems kombinieren, indem man das ausgangssprachliche Lexem und die definitorische Beschreibung zusammenstellt, und somit den Effekt erzielt, dass der Rezipient informiert ist und die Prise Exotik und Fremdheit, was als ein effektives Werbemittel betrachtet werden kann, beibehalten wird.

In Anbetracht der dargestellten Übersetzungsversuche soll nochmals die pragmatische Funktion der Speisebezeichnungen hervorgehoben werden. Im Hinblick auf die Pragmatik soll der Name einerseits „appetitlich“ sein, andererseits soll er etwas kommunizieren. Bei der Übersetzung in Form von Wortübernahme oder Lehnübersetzung bleiben die zwei Bedingungen eher nicht erfüllt.

\section{Literatur}

ARMALYTĖ, Olimpija (1986): Lietuvišku žodžių realijų vertimas ị anglu kalbą: įvadas, pratybu užduotys ir metodiniai nurodymai.Vilnius.

BARLÖSIUS, Eva/NAUMANN, Gerhard/TEUTEBERG, Hans Jürgen (1997): Leitgedanken über die Zusammenhänge von Identität und kulinarischer Kultur im Europa der Regionen. In: Teuteberg, Hans, Jürgen/Neumann, Gerhard/Wierlacher, Alois/Barlösius, Eva (Hg.): Essen und kulturelle Identität. Berlin, S. 13-23.

CHOJNOWSKI, Przemysław (2001): Kulturen im Spiegel der Lexikographie. Polnischdeutsche Wörterbücher als Hilfsmittel bei der Übersetzung kultureller Schlüsselwörter. In: Schatte, Christoph (Hg.): Linguistische und didaktische Probleme der Translatorik. Poznań, S. 21-38.

DĄBROWSKA, Anna (1998): 0 językowym zachowaniu się przy stole. Dlaczego upiększamy nazwy potraw? In: Kowalski, Piotr (Hg.): Oczywisty urok biesiadowania. Wrocław, S. 248-253.

DUCH, Karl (2003): Leksykon sztuki kulinarnej. Międzynarodowa kuchnia hotelowa i restauracyjna. Kraków.

FEYRER, Cornelia (2009): Kultur und Kulinaria im Transfer: Von der Alchemie des Kochens zur Synergie kulinarischer Kultureme in der Translation. In: Lavric, Eva/ Konzett, Carmen (Hg.): Food and Language. Sprache und Essen (=Innsbrucker Beiträge zu Sprache, Kultur und Translation), Bd. 2. Frankfurt/M., S. 389-404.

GERCKEN, Jürgen (1999): Kultur, Sprache und Text als Aspekte von Original und Übersetzung. Frankfurt amMain.

HEJWOWSKI, Krzysztof (2004). Kognitywno-komunikacyjna teoria przekładu. Warszawa.

HOLZER, Peter J. (2009): Pragmatische und kulturpaarspezifische Aspekte der Übersetzung von landestypischen Kulinaria. In: Lavric, Eva/Konzett, Carmen (Hg.): 
Food and Language. Sprache und Essen (=Innsbrucker Beiträge zu Sprache, Kultur und Translation), Bd. 2.Frankfurt/M., S. 377-387.

KOHRS, Jurgita (2008): Litauische Realien auf deutschsprachigen Internetseiten über Litauen. In: Sociolingvistika 12, S. 57-65.

KOLLER, Werner (1994): Einführung in die Übersetzungswissenschaft. Wiesbaden. KOLLER, Werner (2004): Einführung in die Übersetzungswissenschaft. Wiebelsheim. KUJAMÄKI, Pekka (1998): Deutsche Stimmen der Sieben Brüder. Ideologie, Poetik undFunktionen literarischer Übersetzung. Frankfurt/M..

KUJAMÄKI, Pekka (2004): Übersetzung von Realienbezeichnungen in literarischen Texten.In: Kittel, Harald/Frank, Armin Paul/Greiner, Norbert/Hermas, Theo/ Lambert, José/Fritz, Paul (Hg.): Übersetzung - Translation - Traduction. Ein internationales Handbuch zur Übersetzungsforschung. Berlin/New York, S. 920925.

LEPENIES, Wolf (1997): Die Übersetzbarkeit der Kulturen. Ein europäisches Problem, eine Chance für Europa. In: Haverkamp, Anselm (Hg.): Die Sprache der anderen. Übersetzungspolitik zwischen den Kulturen. Frankfurt/M., S. 95-117.

MANNOVÁ, Elena (2007): Stereotypen auf dem Teller. Eine Analyse der Speisenamen in slowakischen Kochbüchern im 20. Jahrhundert. In: Hahn, Hans-Henning/ Mannová, Elena (Hg.): Nationale Wahrnehmung und ihre Stereotypisierung. Beiträge zur Historischen Stereotypenforschung. Frankurt am Main/Berlin/Bern/ Bruxelles/New York/Oxford/Wien, S. 39-58.

MĘDELSKA, Jolanta/ WAWRZYŃCZYK, Jan (1992): Między oryginałem a przekładem. Rzecz o słownikach dwujęzycznych. Kielce.

NEUMANN, Gerhard (1993): „Jede Nahrung ist ein Symbol“. Umrisse einer Kulturwissenschaft des Essens. In: Teuteberg, Hans Jürgen/Neumann, Gerhard/Wierlacher, Alois/Barlösius, Eva (Hg.): Essen und kulturelle Identität.Berlin, S. 385-444.

PIPREK, Jan/ IPPOLDT, Juliusz (1987): Wielki słownik niemiecko-polski. T. 1-2. Warszawa.

PONS (2007): Wielki słownik niemiecko-polski. Poznań.

SCHIRRMEISTER, Claudia (2000): Bratwurst oder Lachsmousse. Die Symbolik des Essens. Bielefeld.

SIERADZKA, Małgorzata (2011): Cepelia, Kresy, szlachta, kontusz... Kritische Anmerkungen zum Umgang der Studierenden mit den Nachschlagewerken bei der Übersetzung von Realienbezeichnungen. In: Bohušová, Zuzanna/Hut'ková, Anita/Małgorzewicz, Anna/Szczęk, Joanna (Hg.): Translationswissenschaft und ihre Zusammenhänge 4 (= Studia Translatorica 2), Dresden/Wrocław, S. 171-186.

SNELL-HORNBY, Mary (2008): Übersetzen und Identität im vielsprachigen Europa. Ein kulinarisch-literarisches Intermezzo. In: Kriegs, Hans P./Mayer, Felix (Hg.): Sprachenvielfalt im Kontext der Fachkommunikation, Übersetzung und Fremdsprachenunterricht. Berlin, S. 87-95.

STANASZEK, Maciej (2007): Tłumacz jako kucharz teoretyk, czyli o przekładzie nazw kulinariów austriackich na język polski. In: Szczęsny, Anna/Hejwowski, Krzysztof (Hg.): Językowy obraz świata w oryginale i przekładzie. Siedlce, S. 449-466. 
SZCZĘK, Joanna/KAŁASZNIK, Marcelina (2012): Wie macht man einen Namen in der Kochkunst - Zur Analyse der Nominationsprozesse im Kulinarischen (am Beispiel der Kochrezepte für Eisdesserts). In: Zbornik za jezik i književnost Filozofskog fakulteta u Novom Sadu, Vol. 2, S. 123-137.

SZCZĘK, Joanna/KAŁASZNIK, Marcelina: ,Utracone $w$ tłumaczeniu' - o problemach $z$ tłumaczeniem nazw potraw z języka polskiego na język niemiecki (im Druck).

THOME, Giesela (2004): Typologie von Übersetzungsschwierigkeiten aus sprachwissenschaftlicher Perspektive. In: Kittel, Harald/House, Juliane/Schultze, Brigitte (Hg.): Traduction: encyclopédie internationale de la recherchesur la traduction. Berlin,S. 436-446.

TSCHOFEN, Bernhard (2008): Kulinaristik und Regionalkultur. In: Wierlacher, Alois/ Bendix, Regina (Hg.): Kulinaristik. Forschung - Lehre - Praxis. Berlin, S. 63-78.

VLACHOV, Sergiej/Florin, Sider (1980): Neperevodimoje v perevode. Moskva.

WIERLACHER, Alois (2013): Das Konzept „Kulinaristik”. In: Ernährungsumschau 11/2013, S. 634-641.

WIERZBICKA, Anna (2007): Słowa klucze. Różne języki - różne kultury. Warszawa. WIKTOROWICZ, Józef/FRĄCZEK, Agnieszka(2010): Wielki słownik niemiecko-polski. Warszawa.

WITASZEK-SAMBORSKA, Małgorzata (2005): Studia nad słownictwem kulinarnym we wspótczesnej polszczyźnie. Poznań.

ŻARSKI, Waldemar (2003): Nazwy zup w języku polskim. In: Rozprawy Komisji Językowej Wrocławskiego Towarzystwa Naukowego XXIX, S. 157-161.

\section{Internetquellen}

http://www.golocal.de/muenchen/oktoberfest/bayerische-spezialitaeten/geschichte/, Zugriff am 15.05.2014.

http://www.kulinarischer-stadtrundgang.de/typisch-deutsches-essen/, Zugriff am 15.05.2014.

http://www.rezepte-und-tipps.de/Deutsche_Rezepte/, Zugriff am 15.05.2014. http://www.deutsche-lebensart.de/41.html, Zugriff am 15.05.2014. http://www.chefkoch.de/magazin/artikel/2062,0/Chefkoch/Deutsche-Kueche-diese-Rezepte-sind-typisch-deutsch.html, Zugriff am 15.05.2014.

http://www.alumniportal-deutschland.org/deutschland/essen-trinken/artikel/ spezialitaeten-regionale-spezialitaeten-deutsche-kueche-deutsche-spezialitaeten.html, Zugriff am 15.05.2014.

http://deutschkochen.blogspot.com/p/die-liste.html, Zugriff am 15.05.2014. http://www.austria.info/at/kulinarik-in-oesterreich/die-oesterreichische-kueche-1088974.html, Zugriff am 15.05.2014.

http://typischich.at/home/wienerin/geniessen/1269940/10-Rezepte_Osterreichische-Kuche, Zugriff am 15.05.2014.

http://oesterreich.pbworks.com/w/page/6235055/\%C3\%96sterreichische\%20 $\mathrm{K} \% \mathrm{C} 3 \% \mathrm{BCche}$ 
http://www.schweizerseiten.ch/rezepte/rezepte.htm, Zugriff am 15.05.2014.

http://www.helpster.de/typisch-schweiz-so-lernen-sie-die-besonderheiten-des-

landes-, Zugriff am 15.05.2014.

http://www.essen-und-trinken.de/schweizer-kueche/schweizer-kueche-1017775.

html, Zugriff am 15.05.2014.

http://iinspiracje-kulinarne.blogspot.com/2013/12/currywurst-cos-z-kuchni-niemieckiej.html, Zugriff am 15.05.2014.

http://www.przyslijprzepis.pl/przepis/currywurst-czyli-niemieckie-kielbaskie-z-

curry, Zugriff am 15.05.2014. http://www.gotowanie.v9.pl/slownik/66.php, Zugriff am 15.05.2014. http://ugotuj.to/ugotuj/-1797869/zupa+eintopf+po+w ielkopolsku/p/, Zugriff am 15.05.2014.http://smaker.pl/polecane/Eintopf, Zugriff am 15.05.2014.

http://www.facetnatalerzu.pl/2012/03/niebo-i-ziemia-czyli-himmel-und-erde. html, Zugriff am 15.05.2014.

http://kwiecienkaodkuchni.blox.pl/2011/02/Kalter-Hund.html, Zugriff am 15.05.2014.

http://zmiksowani.pl/przepisy-na/kasseler-pieczony-schab-wedzony, Zugriff am 15.05.2014.

http://www.forumowisko.pl/topic/54173-niemiecki/, Zugriff am 15.05.2014. http://www.magicwindow.internetdsl.pl/dok/niemcy.pdf, Zugriff am 15.05.2014. http://bistro24.pl/gotowanie/przepisy/kotlety-wedzone-kassler-w-glazurzemiodowej,116819.html, Zugriff am 15.05.2014.

http://www.grupy.senior.pl/Re-OT-Nazewnictwo-kulinarne,p,2318462,f,1,9.html, Zugriff am 15.05.2014.

http://barbaramariawkuchni.blogspot.com/2013/01/szynka-kassler-z-zapiekan-

ka-ziemniaczana.html, Zugriff am 15.05.2014.

http://durszlak.pl/przepisy-kulinarne/klopsy-w-sosie, Zugriff am 15.05.2014.

http://allrecipes.pl/przepis/3769/bawarska-piecze--z-marynowanego-mi-sa--sau-

erbraten-.aspx, Zugriff am 15.05.2014.

http://hasla.blox.pl/Blox/szukaj/kluski+z+ziemniakami, Zugriff am 15.05.2014.

http://zmiksowani.pl/przepis,567900,kluski-ziemniaczane-badische-schupfnudeln.html, Zugriff am 15.05.2014.

http://kobieta.interia.pl/kuchnia/makarony/news-schupfnudeln-niemieckie-kluski-ziemniaczane,nId,709211, Zugriff am 15.05.2014.

http://www.facetnatalerzu.pl/2012/01/spatzle-szpecle-maczne-kluseczki.html, Zugriff am 15.05.2014.

http://www.pieceofcake.pl/Przepisy/Zacierka, Zugriff am 15.05.2014.

http://www.mojewypiekiinietylko.com/2014/07/szpecle-spatzle/, Zugriff am 15.05.2014.

http://www.facetnatalerzu.pl/2012/01/spatzle-szpecle-maczne-kluseczki.html, Zugriff am 15.05.2014. 